DOI: $10.6060 / \mathrm{mhc} 2012.110736 \mathrm{~b}$

\title{
Catalytic Effect of Thioles in Oxidation of the Vinyl Group to Aldehyde in Chlorophyll $a$ Derivatives
}

\author{
Dmitry V. Belykh, @ and Ekaterina V. Ashikhmina \\ Institute of Chemistry, Komi Scientific Center, Russian Academy of Sciences, 167982 Syktyvkar, Russia \\ @Corresponding authorE-mail: belykh-dv@chemi.komisc.ru,belykh-dv@mail.ru
}

\begin{abstract}
It is shown that the vinyl group of chlorophyll a derivatives (methylpheophorbide a, methylpyropheophorbide a, 13-amides of chlorin $e_{0}$ can be oxidized to the aldehyde under mild conditions by atmospheric oxygen in the presence of thiols as catalysts. The corresponding aldehyde can be obtained in 50-55\% yields. The possible mechanism of the catalytic action of thiols in the oxidation of the vinyl group is proposed.
\end{abstract}

Keywords: Chlorophyll $a$, methylpheophorbide $a$, methylpyropheophorbide $a$, chlorin $e_{6}$, oxidation, thiols, catalysis.

Interest in the chemical transformations of chlorophylls and their derivatives is due not only to significant role of these compounds in nature, but also the possibility of their use as precursors for the synthesis of compounds, which find application in various fields (medicine, ${ }^{[1-3]}$ catalysis ${ }^{[4]}$ design of polymeric nanomaterials for photoelectronics, ${ }^{\left[{ }^{[5-8]}\right.}$ etc.) Oxidation of the vinyl group in chlorophyll $a$ and its derivatives to the aldehyde with formation of the corresponding $d$-series derivatives is widely used in the chemistry of these compounds as an initial conversion step. The aldehyde group in the position 3 can be further involved in a variety of reactions. The literature describes the chemical modification of the $d$-series derivatives using the reductive amination, ${ }^{[9,10]}$ Wittig, ${ }^{[11]}$ McMurray, ${ }^{[12,13]}$ and Knoevenage ${ }^{[11]}$ reactions. Reactions of the aldehyde group of $d$-chlorins were used to form fluoroacy ${ }^{[14]}$ and ethenyl ${ }^{[15]}$ substituents. Formylchlorins, obtained by oxidation of the vinyl group of chlorophyll $a$ derivatives, can be used as aromatic aldehydes for formation of any porphyrin macrocycle in the synthesis of various dimeric porphyrins. ${ }^{[16,17]}$ Oxidation of the vinyl group of chlorophyll $a$ derivatives are carried out in most cases by reductive ozonolysis ${ }^{[11,18,19]}$ or using osmium compounds, mainly, catalytically, when diol, formed in situ by the action of osmium tetraoxide in catalytic amounts, is oxidized with sodium periodates (see reviews ${ }^{[18-20]}$ and references therein). Wagner reaction (oxidation by $\mathrm{KMnO}_{4}$ ), used in the conversion of chlorophyll $a$ to chlorophyll $d$ in order to prove the structure of the latter, ${ }^{[21]}$ did not received application in the chemistry of chlorophylls and their derivatives because of low selectivity. Recently it was reported that when the various thiols react with methylpyropheophorbide $a$ in the presence of air, instead of the expected addition of thiol to vinyl group, the various oxidation processes take place, including oxidation of the vinyl group to an aldehyde. ${ }^{[22]}$ Oxidation of the vinyl group, according to ${ }^{[22]}$, occurs in the presence of five-fold excess of thiol and four-fold excess of $p$-toluenesulfonic acid. A similar oxidation, we have also observed when tried to carry out the addition reaction with a number of thiols with participation of some derivatives of chlorophyll $a(\mathbf{1 - 5})$ (Scheme 1).

Here we have studied in more detail the oxidation of chlorophyll $a$ derivatives (1-5) by atmospheric oxygen in the presence of different thiols ${ }^{\#}$ (Scheme 1). It was found that thiols can catalyze the oxidation of the vinyl group to the aldehyde by air oxygen. To receive the target aldehydes (6-10) it is enough to use catalytic amounts of thiol (only 5-7 molar \% respectively the amount of oxidized vinylchlorin). Oxidation was carried out by bubbling of air through a solution of vinylchlorin at room temperature for 1 h. The optimal oxidation conditions were worked out using methylpheophorbide $a(\mathbf{1})$ as an oxidation substrate, different thiols (see Scheme 1) and various solvents (benzene, $\mathrm{CHCl}_{3}$, $\mathrm{CH}_{2} \mathrm{Cl}_{2}$ and THF). The best results in terms of yield of the desired aldehyde (6) (50-55\%) were obtained when the reaction was carried out in the THF using thiohexanol-1 as a catalyst. The removal of a thiol causes some problems in the treatment of reaction mixture after completion of the reaction. Using of thiohexanol-1 is the most convenient in terms of removing it from the reaction mixture, which can be carried out by three to fourfold recrystallization from a mixture of chloroform with methanol or methylene chloride with hexane. Oxidation of other chlorins (2-5) can be carried out under similar conditions (THF, 5-7 molar \% of hexanethiol-1, air bubbling for 1 hour), the yields of the corresponding aldehydes (7-10) being the same as in the case of oxidation of 1. Decreasing of the yield was not observed even in the case of methypheophorbide $a$ (2) bearing an easily oxidizable exocycle.

Certain assumptions about how the thiols may be involved in the oxidation of the vinyl group of methylpheophorbide $a$ were expressed in ${ }^{[22]}$, but all the reactions that lead to the formation of an aldehyde were not presented. Taking into account literature data ${ }^{[15]}$ and our own results, it is necessary to note that the proposed mechanism should, firstly, specify the pathways of the double bond breaking between $\mathrm{C}\left(3^{1}\right)$ and $\mathrm{C}\left(3^{2}\right)$, and, secondly, explain, how the regeneration of thiol proceeds to make it catalytically active. Proposed mechanism of the catalytic action of thiols in the oxidation of vinyl group of chlorophyll $a$ derivatives by $\mathrm{O}_{2}$ is shown in Scheme 1. We assume that on the first stage the vinyl group of chlorin interacts with the radical RS; which is formed from the initial thiol under action of oxygen, to form an intermediate free radical I. It is known 


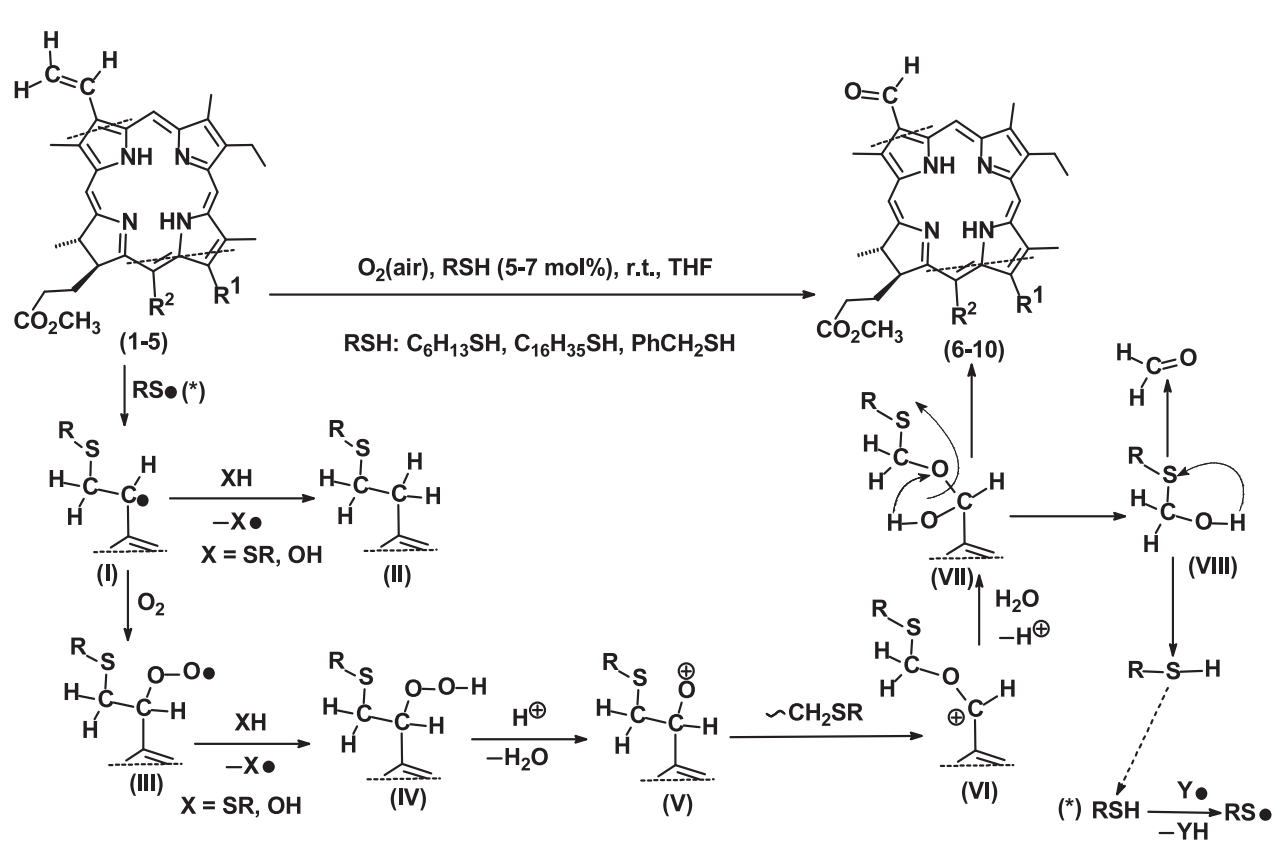

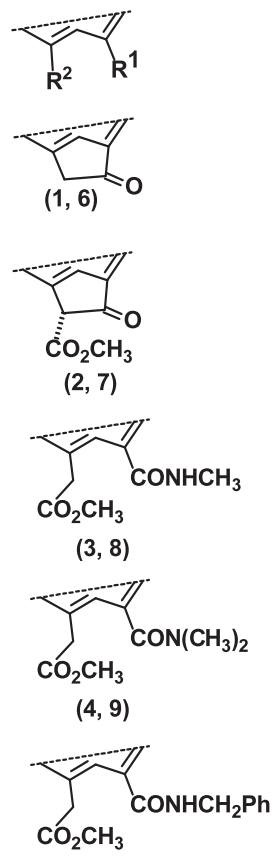

$(5,10)$

Scheme 1.

that $A_{R}$ addition of thiols to alkenes is frequently accompanied by oxidation of hydrocarbon substituents as a side reaction. ${ }^{[23]}$ In order to confirm the possibility of $A_{R}$ reaction in the oxidative conditions the interaction of equimolar amounts of methylpheophorbide $a$ (2) with thiohexanol-1 was studied without oxygen (under argon). The addition reaction product (II), forming according to $A_{R}$ mechanism, was identified by the presence in the mass spectrum of the resulting reaction mixture of the corresponding molecular ion peak $[\mathrm{M}+\mathrm{H}]^{+}$with $m / z$ 725.3. Since in the conditions of oxidation the $A_{R}$ reaction may proceed, then all the other alternative reactions that have the same stages are also possible. For example, the interaction of radical I with oxygen results in the formation of hydroperoxide (Scheme 1, intermediate IV, forming through the hydroperoxide radical III). Similar processes were described earlier, ${ }^{[i . e .23]}$ the same assumption was made in ${ }^{[22]}$. It is known, that in the presence of acid the water molecules may split off from the protonated molecule of hydroperoxide with following rearrangement of the resulting cation with formation of hemiacetal, which is then spontaneously decomposed to the corresponding carbonyl compound. Such a mechanism is proposed, i.e. for the decomposition of cumyl hydroperoxide. ${ }^{[24]}$ Similar processes may occur in the case of intermediate hydroperoxide IV (Scheme 1), presumably formed by the reaction of thiols with chlorophyll $a$ derivatives in the presence of oxygen. Thiol molecule can act as the proton donor. Cation $\mathbf{V}$, forming as a result of water molecule splitting, can spontaneously rearranged due to migration of alkylthiomethyl fragment with the formation of carbocation VI (Scheme 1). After the interaction of carbocation VI with a water molecule the hemiacetal VII is formed, the decomposition of which gives hemithioacetal VIII and corresponding formylchlorin. Spontaneous decomposition of hemithioacetal VIII leads to the formation of the initial thiol and formaldehyde (Scheme 1). As a result, regenera- tion of the catalyst takes place, which can react with the new molecule of vinylchlorin.

Thus, we have shown that thiols can be catalysts for the oxidation of the vinyl group of chlorophyll $a$ derivatives to an aldehyde and proposed an explanation of the catalytic action of thiols in this reaction. Catalytic effect of thiols can be explained by the intermediate formation of hydroperoxides in the interaction of vinylchlorin with thiol and oxygen, the following spontaneous conversion of which leads to the formation of formylchlorin and regeneration of thiol.

\section{Notes and References}

\# $\quad{ }^{1} \mathrm{H}$ NMR spectra were recorded in $\mathrm{CDCl}_{3}$ on spectrometer Bruker Avance II (working frequency $300 \mathrm{MHz}, \mathrm{CDCl}_{3}$ ). UV-Vis spectra were recorded on spectrometer Shimadzu UV-1700 (PharmaSpec) in $\mathrm{CHCl}_{3}$ in $200-1100 \mathrm{~nm}$ range in $10 \mathrm{~mm}$ quartz cuvettes, using $\mathrm{CHCl}_{3}$ as comparison sample. Monitoring the reaction proceeding was performed by TLC on Silufol plates, eluent $-\mathrm{CCl}_{4}$-acetone (4:1 vol). Methylpheophorbide $a$ (2), ${ }^{[25]}$ methylpyropheophorbide $a(1),{ }^{[26]}$ methylamide (3) and dimethylamide (4), ${ }^{[27]}$ benzylamide (5) ${ }^{[28]}$ were obtained according to the known procedures. To carry out the oxidation in solution the air was bubbled (flow rate 4-5 $\mathrm{l} / \mathrm{h}$ ) through the solution of chlorophyll $a$ derivative (30-40 $\mathrm{mg}$ ) and 5-7 $\mathrm{mol} \%$ hexanethiol in THF $(40-50 \mathrm{ml})$ at r.t. After the reaction complete THF was evaporated, the residue after evaporation was recrystallized from a mixture of chloroform and methanol or methylene chloride and hexane to obtain the powder mass. Final purification of formylchlorin was performed by column chromatography on silica gel as in ${ }^{[29,30]}$. The spectral characteristic ${ }^{[31]}$ of the obtained formylchlorins (6-10) are identical to described earlier. ${ }^{[29,30]}$

" Methylpheophorbide d (6). ${ }^{1} \mathrm{H}$ NMR $\delta$ ppm: $11.50 \mathrm{~s}(1 \mathrm{H}, 3-\mathrm{CH}=\mathrm{O})$, $10.21 \mathrm{~s}\left(1 \mathrm{H}, \mathrm{H}^{5}\right), 9.53 \mathrm{~s}\left(1 \mathrm{H}, \mathrm{H}^{10}\right), 8.82 \mathrm{~s}\left(1 \mathrm{H}, \mathrm{H}^{20}\right), 5.34 \mathrm{~d}(1 \mathrm{H}$, $\left.\mathrm{H}^{13(2)}{ }_{\mathrm{A}}, J=20.0 \mathrm{~Hz}\right), 5.18 \mathrm{~d}\left(1 \mathrm{H}, \mathrm{H}^{13(2)}, J=20.0 \mathrm{~Hz}\right), 4.56 \mathrm{qd}(1 \mathrm{H}$, $\mathrm{H}^{18}, \stackrel{{ }^{A}}{J} 7.3$ and $\left.1.9 \mathrm{~Hz}\right), 4.38 \mathrm{~m}\left(1 \mathrm{H}, \mathrm{H}^{17}\right), 3.66 \mathrm{q}\left[2 \mathrm{H}, 8-\left(\mathrm{CH}_{2} \mathrm{CH}_{3}\right)\right.$, $J=8.1 \mathrm{~Hz}], 3.75 \mathrm{~s}\left[3 \mathrm{H}, 17-\left(\mathrm{CH}_{2} \mathrm{CH}_{2} \mathrm{COOCH}_{3}\right)\right], 3.68 \mathrm{~s}(3 \mathrm{H}, 12-$ $\left.\mathrm{CH}_{3}\right), 3.62 \mathrm{~s}\left(3 \mathrm{H}, 2-\mathrm{CH}_{3}\right), 3.26 \mathrm{~s}\left(3 \mathrm{H}, 7-\mathrm{CH}_{3}\right), 2.75-2.27 \mathrm{~m}[4 \mathrm{H}$, 
$\left.17-\left(\mathrm{CH}_{2} \mathrm{CH}_{2} \mathrm{COOCH}_{3}\right)\right], 1.85 \mathrm{~d}\left(3 \mathrm{H}, 18-\mathrm{CH}_{3}, J=6.8 \mathrm{~Hz}\right), 1.68 \mathrm{t}(3 \mathrm{H}$, $8-\mathrm{CH}_{2} \mathrm{CH}_{3}, J=7.8 \mathrm{~Hz}$ ), -0.38 brs (1H, I-NH), -2.18 brs ( $\left.1 \mathrm{H}, \mathrm{III}-\mathrm{NH}\right)$. Methylpheophorbide d (7). 'H NMR $\delta$ ppm: $11.49 \mathrm{~s}(1 \mathrm{H}, 3-\mathrm{CH}=\mathrm{O})$, $10.16 \mathrm{~s}\left(1 \mathrm{H}, \mathrm{H}^{5}\right), 9.55 \mathrm{~s}\left(1 \mathrm{H}, \mathrm{H}^{10}\right), 8.83 \mathrm{~s}\left(1 \mathrm{H}, \mathrm{H}^{20}\right), 6.33 \mathrm{~s}\left(1 \mathrm{H}, \mathrm{H}^{13(2)}\right)$, $4.53 \mathrm{qd}\left(1 \mathrm{H}, \mathrm{H}^{18}, J=7.3\right.$ and $\left.2.0 \mathrm{~Hz}\right), 4.25 \mathrm{dt}\left(1 \mathrm{H}, \mathrm{H}^{17}, J=8.0\right.$ and 2.2 $\mathrm{Hz}), 3.91 \mathrm{~s}\left[3 \mathrm{H}, 13(2)-\left(\mathrm{COOCH}_{3}\right)\right], 3.67-3.61 \mathrm{~m}\left[2 \mathrm{H}, 8-\left(\mathrm{CH}_{2} \mathrm{CH}_{3}\right)\right.$, $J=8.1 \mathrm{~Hz}], 3.71 \mathrm{~s}\left(3 \mathrm{H}, 7-\mathrm{CH}_{3}\right), 3.70 \mathrm{~s}\left(3 \mathrm{H}, 2-\mathrm{CH}_{3}\right), 3.58 \mathrm{~s}[3 \mathrm{H}$, $\left.17-\left(\mathrm{CH}_{2} \mathrm{CH}_{2} \mathrm{COOCH}_{3}\right)\right], 3.23 \mathrm{~s}\left(3 \mathrm{H}, 12-\mathrm{CH}_{3}\right), 2.73-2.50 \mathrm{~m}[2 \mathrm{H}, 17-$ $\left.\left(\mathrm{CH}_{2} \mathrm{CH}_{2} \mathrm{COOCH}_{3}\right)\right], 2.37-2.21 \mathrm{~m}\left[2 \mathrm{H}, 17-\left(\mathrm{CH}_{2} \mathrm{CH}_{2} \mathrm{COOCH}_{3}\right)\right], 1.85$ $\mathrm{d}\left(3 \mathrm{H}, 18-\mathrm{CH}_{3}, J=6.8 \mathrm{~Hz}\right), 1.68 \mathrm{t}\left(3 \mathrm{H}, 8-\mathrm{CH}_{2} \mathrm{CH}_{3}, J=7.8 \mathrm{~Hz}\right),-0.14$ br.s (1H, I-NH), -2.10 br.s (1H, III-NH).

Chlorin e 3-desvinyl-3-formyl-13(1)-N-(methyl)amide-15(2), 17(3) dimethyl ester (8). ${ }^{1} \mathrm{H}$ NMR $\delta$ ppm: $11.50 \mathrm{~s}(1 \mathrm{H}, 3-\mathrm{CH}=\mathrm{O})$, $10.27 \mathrm{~s}\left(1 \mathrm{H}, \mathrm{H}^{5}\right), 9.62 \mathrm{~s}\left(1 \mathrm{H}, \mathrm{H}^{10}\right), 8.96 \mathrm{~s}\left(1 \mathrm{H}, \mathrm{H}^{20}\right), 6.41 \mathrm{~m}(1 \mathrm{H}, 13-$ $\mathrm{CONHCH}_{3}$ (amide), $J=4.0 \mathrm{~Hz}$ ), $5.56 \mathrm{~d}\left(1 \mathrm{H}, \mathrm{H}^{15(1)}, J=20.0 \mathrm{~Hz}\right)$, $5.29 \mathrm{~d}\left(1 \mathrm{H}, \mathrm{H}^{15(1)}{ }_{\mathrm{B}}, J=20.0 \mathrm{~Hz}\right), 4.48 \mathrm{q}\left(1 \mathrm{H}, \mathrm{H}^{18}, J=7.0 \mathrm{~Hz}\right), 4.36$ br.s $\left(1 \mathrm{H}, \mathrm{H}^{17}, J=9.2 \mathrm{~Hz}\right), 3.82 \mathrm{~s}\left(3 \mathrm{H}, 15-\mathrm{CH}_{2} \mathrm{CO}_{2} \mathrm{CH}_{3}\right), 3.78 \mathrm{~s}(3 \mathrm{H}$, $\left.2-\mathrm{CH}_{3}\right), 3.65 \mathrm{~s}\left(3 \mathrm{H}, 17-\mathrm{CH}_{2} \mathrm{CH}_{2} \mathrm{CO}_{2} \mathrm{CH}_{3}\right), 3.55 \mathrm{~s}\left(3 \mathrm{H}, 12-\mathrm{CH}_{3}\right), 3.30$ $\mathrm{s}\left(3 \mathrm{H}, 7-\mathrm{CH}_{3}\right), 3.29 \mathrm{~d}\left(3 \mathrm{H}, 13-\mathrm{CONHCH}_{3}, J=4.8 \mathrm{~Hz}\right), 3.74 \mathrm{q}(2 \mathrm{H}$, $\left.8-\mathrm{CH}_{2} \mathrm{CH}_{3}, J=7.6 \mathrm{~Hz}\right), 2.0-2.6 \mathrm{~m}\left(4 \mathrm{H}, 17-\mathrm{CH}_{2} \mathrm{CH}_{2} \mathrm{CO}_{2} \mathrm{CH}_{3}\right), 1.73$ d $\left(3 \mathrm{H}, 18-\mathrm{CH}_{3}, J=7.0\right), 1.70 \mathrm{t}\left(3 \mathrm{H}, 8-\mathrm{CH}_{2} \mathrm{CH}_{3}, J=7.7 \mathrm{~Hz}\right),-1.50$ br.s (1H, I-NH), -2.00 br.s (1H, III-NH).

Chlorin e 3-desvinyl-3-formyl-13(1)-N, N-(dimethyl)amide15(2), 17(3) dimethyl ester (9). ' $\mathrm{H}$ NMR $\delta$ ppm: Predominant isomer: $11.57 \mathrm{~s}(1 \mathrm{H}, 3-\mathrm{CH}=\mathrm{O}), 10.34 \mathrm{~s}\left(1 \mathrm{H}, \mathrm{H}^{5}\right), 9.66 \mathrm{~s}\left(1 \mathrm{H}, \mathrm{H}^{10}\right), 9.00 \mathrm{~s}(1 \mathrm{H}$, $\left.\mathrm{H}^{20}\right), 5.87 \mathrm{~d}\left(1 \mathrm{H}, \mathrm{H}^{15(1)}{ }_{\mathrm{A}}, J=18.7 \mathrm{~Hz}\right), 5.08 \mathrm{~d}\left(1 \mathrm{H}, \mathrm{H}^{15(1)}{ }_{\mathrm{B}}, J=18.7\right.$ $\mathrm{Hz}), 4.50 \mathrm{q}\left(1 \mathrm{H}, \mathrm{H}^{18}, J=7.0 \mathrm{~Hz}\right), 4.40$ br.s $\left(1 \mathrm{H}, \mathrm{H}^{17}, J=9.6 \mathrm{~Hz}\right)$, $3.80 \mathrm{~m}\left(2 \mathrm{H}, 8-\mathrm{CH}_{2} \mathrm{CH}_{3}\right), 3.82 \mathrm{~s}\left(3 \mathrm{H}, 15-\mathrm{CH}_{2} \mathrm{CO}_{2} \mathrm{CH}_{3}\right), 3.81 \mathrm{~s}(3 \mathrm{H}$, $\left.2-\mathrm{CH}_{3}\right), 3.67 \mathrm{~s}\left(3 \mathrm{H}, 17-\mathrm{CH}_{2} \mathrm{CH}_{2} \mathrm{CO}_{2} \mathrm{CH}_{3}\right), 3.50 \mathrm{~s}\left(3 \mathrm{H}, 12-\mathrm{CH}_{3}\right)$, $3.47 \mathrm{~s}\left(3 \mathrm{H}, 13-\mathrm{CONCH}_{3} \mathrm{CH}_{3}\right), 3.36 \mathrm{~s}\left(3 \mathrm{H}, 7-\mathrm{CH}_{3}\right), 2.77 \mathrm{~s}[3 \mathrm{H}$, $\left.13-\mathrm{CON}\left(\mathrm{CH}_{3}\right)\left(\mathrm{CH}_{3}\right)\right], 2.0-2.6 \mathrm{~m}\left(4 \mathrm{H}, 17-\mathrm{CH}_{2} \mathrm{CH}_{2} \mathrm{CO}_{2} \mathrm{CH}_{3}\right), 1.60-$ $1.80 \mathrm{~m}\left(6 \mathrm{H}, 18-\mathrm{CH}_{3}, 8-\mathrm{CH}_{2} \mathrm{CH}_{3}\right),-1.48$ br.s $(1 \mathrm{H}, \mathrm{I}-\mathrm{NH}),-2.00$ br.s $(1 \mathrm{H}, \mathrm{III}-\mathrm{NH})$. Minor isomer: $11.56 \mathrm{~s}(1 \mathrm{H}, 3-\mathrm{CH}=\mathrm{O}), 10.34 \mathrm{~s}(1 \mathrm{H}$, $\left.\mathrm{H}^{5}\right), 9.66 \mathrm{~s}\left(1 \mathrm{H}, \mathrm{H}^{10}\right), 8.97 \mathrm{~s}\left(1 \mathrm{H}, \mathrm{H}^{20}\right), 5.66 \mathrm{~d}\left(1 \mathrm{H}, \mathrm{H}^{15(1)}{ }_{\mathrm{A}}, J=19.3\right.$ $\mathrm{Hz}), 5.16 \mathrm{~d}\left(1 \mathrm{H}, \mathrm{H}^{15(1)}{ }_{\mathrm{B}}, J=19.3 \mathrm{~Hz}\right), 4.50 \mathrm{q}\left(1 \mathrm{H}, \mathrm{H}^{18}, J=7.0\right.$ $\mathrm{Hz}), 4.45$ br.s $\left(1 \mathrm{H}, \mathrm{H}^{17}, J=9.4 \mathrm{~Hz}\right), 3.80 \mathrm{~m}\left(2 \mathrm{H}, 8-\mathrm{CH}_{2} \mathrm{CH}_{3}\right)$, $3.81 \mathrm{~s}\left(3 \mathrm{H}, 2-\mathrm{CH}_{3}\right), 3.79 \mathrm{~s}\left(3 \mathrm{H}, 15-\mathrm{CH}_{2} \mathrm{CO}_{2} \mathrm{CH}_{3}\right), 3.64 \mathrm{~s}(3 \mathrm{H}, 17-$ $\left.\mathrm{CH}_{2} \mathrm{CH}_{2} \mathrm{CO}_{2} \mathrm{CH}_{3}\right), 3.51 \mathrm{~s}\left[3 \mathrm{H}, 13-\mathrm{CON}\left(\mathrm{CH}_{3}\right)\left(\mathrm{CH}_{3}\right)\right], 3.49 \mathrm{~s}(3 \mathrm{H}$, $\left.12-\mathrm{CH}_{3}\right), 3.36 \mathrm{~s}\left(3 \mathrm{H}, 7-\mathrm{CH}_{3}\right), 3.14 \mathrm{~s}\left[3 \mathrm{H}, 13-\mathrm{CON}\left(\mathrm{CH}_{3}\right)\left(\mathrm{CH}_{3}\right)\right]$, 2.0-2.6 m (4H, 17- $\left.\mathrm{CH}_{2} \mathrm{CH}_{2} \mathrm{CO}_{2} \mathrm{CH}_{3}\right), 1.60-1.80 \mathrm{~m}\left(6 \mathrm{H}, 18-\mathrm{CH}_{3}\right.$, $\left.8-\mathrm{CH}_{2} \mathrm{CH}_{3}\right),-1.48$ br.s $(1 \mathrm{H}, \mathrm{I}-\mathrm{NH}),-1.86$ br.s $(1 \mathrm{H}, \mathrm{III}-\mathrm{NH})$.

Chlorin e 3-desvinyl-3-formyl-13(1)-N-(benzyl)amide-15(2), 17(3)-dimethyl ester (10). ${ }^{1} \mathrm{H}$ NMR $\delta$ ppm: 11.52 s $(1 \mathrm{H}$, $3-\mathrm{CH}=\mathrm{O}), 10.26 \mathrm{~s}\left(1 \mathrm{H}, \mathrm{H}^{5}\right), 9.63 \mathrm{~s}\left(1 \mathrm{H}, \mathrm{H}^{10}\right), 8.90 \mathrm{~s}\left(1 \mathrm{H}, \mathrm{H}^{20}\right)$, $7.59 \mathrm{~d}\left(2 \mathrm{H}, \quad 13-\mathrm{CONHCH}_{2} P h\right.$ (ortho-H), $\left.J=7.9 \mathrm{~Hz}\right), 7.46$ t $\left(2 \mathrm{H}, 13-\mathrm{CONHCH}_{2} P h(\right.$ meta-H $\left.), J=7.5 \mathrm{~Hz}\right), 7.37 \mathrm{t}(1 \mathrm{H}$, 13- $\mathrm{CONHCH}_{2}$ Ph $($ para-H), $J=7.5 \mathrm{~Hz}), 6.82$ br.s $(1 \mathrm{H}, 13-$ $\left.\mathrm{CONHCH}_{2} \mathrm{Ph}, J=5.3 \mathrm{~Hz}\right), 5.59 \mathrm{~d}\left(1 \mathrm{H}, \mathrm{H}^{15(1)}{ }_{\mathrm{A}}, J=19.0 \mathrm{~Hz}\right)$, and $5.30 \mathrm{~d}\left(1 \mathrm{H}^{2}, \mathrm{H}^{15(1)}{ }_{\mathrm{B}}, J=19.3 \mathrm{~Hz}\right) ; 13-\mathrm{CONHCH}_{2} \mathrm{Ph}: 5.12$ and 4.80 both dd $(1 \mathrm{H} J=14.3$ and $6.1 \mathrm{~Hz}), 4.47 \mathrm{q}\left(1 \mathrm{H}, \mathrm{H}^{18}, J=7.2 \mathrm{~Hz}\right)$, 4.40 br.s $\left(1 \mathrm{H}, \mathrm{H}^{17}, J=9.2 \mathrm{~Hz}\right), 3.78 \mathrm{~s}\left(3 \mathrm{H}, 15-\mathrm{CH}_{2} \mathrm{CO}_{2} \mathrm{CH}_{3}\right), 3.73$ $\mathrm{s}\left(3 \mathrm{H}, 2-\mathrm{CH}_{3}\right), 3.71 \mathrm{q}\left(2 \mathrm{H}, 8-\mathrm{CH}_{2} \mathrm{CH}_{3}, J=7.5 \mathrm{~Hz}\right), 3.63 \mathrm{~s}(3 \mathrm{H}, 17-$ $\left.\mathrm{CH}_{2} \mathrm{CH}_{2} \mathrm{CO}_{2} \mathrm{CH}_{3}\right), 3.52 \mathrm{~s}\left(3 \mathrm{H}, 12-\mathrm{CH}_{3}\right), 3.31 \mathrm{~s}\left(3 \mathrm{H}, 7-\mathrm{CH}_{3}\right), 2.0-2.8$ $\mathrm{m}\left(4 \mathrm{H}, 17(1), 17(2)-\mathrm{CH}_{2}\right), 1.71 \mathrm{~d}\left(3 \mathrm{H}, 18-\mathrm{CH}_{3}, J=7.2 \mathrm{~Hz}\right), 1.67 \mathrm{t}$ $\left(3 \mathrm{H}, 8-\mathrm{CH}_{2} \mathrm{CH}_{3}, J=7.5 \mathrm{~Hz}\right),-1.50$ br.s $(1 \mathrm{H}, \mathrm{I}-\mathrm{NH}),-1.98$ br.s $(1 \mathrm{H}$, III-NH).

1. Nyman E.S., Hynninen P.H. J. Photochem. Photobiol., B: Biology 2004, 73, 1-28.

2. Sternberg E.D., Dolphin D., Brückner Tetrahedron 1998, 54, 4151-4202.
3. Bregadze V.I., Sivaev I.B., Gabel D., Wöhrle D. J. Porphyrins Phthalocyanines 2001, 5, 767-781.

4. Gryglik D, Miller J.S., Ledakowicz S. Solar Energy 2004, 77, 615-623.

5. Amao Y., Komori T. Biosensors and Bioelectronics 2004, 19, 843-847.

6. Amao Y., Yamada Y. Biosensors and Bioelectronics 2007, 22, 1561-1565.

7. Alekseev A.S., Tkachenko N.V., Tauber A.Y., Hynninen P.H., Osterbacka R., Stubb H., Lemmetyinen H. Chem. Phys. 2002, 275, 243-251.

8. Tkachenko N.V., Hynninen P.H., Lemmetyinen H. Chem. Phys. Lett. 1996, 261, 234-240.

9. Davis R.C., Ditson S.L., Fentiman A.F., Pearlstein R.M. J. Am. Chem. Soc. 1981, 103, 6823-6826.

10. Tamiaki H., Holzwarth A.R., Schaffner K. Photosynth. Res. 1994, 41, 245-251.

11. Tamiaki H., Kouraba M. Tetrahedron. 1997, 53, 1067710688.

12. Goswami L.N., Ethirajan M., Dobhal M.P., Zhang M., Missert J.R., Shibata M., Kadish K.M., Pandey R.K. J. Org. Chem. 2009, 74, 568-579.

13. Jaquinod L., Nucro D.J., Medforth C.J., Pandey R.K., Forsyth T.P., Olmstead M.M., Smith K.M. Angew. Chem. Int. Ed. Engl. 1996, 35, 1013-1016.

14. Sasaki S., Kotegawa Y., Tamiaki H. Tetrahedron Lett. 2006, 47, 4849-4852.

15. Sasaki S., Mizutani K., Kunieda M., Tamiaki H. Tetrahedron Lett. 2008, 49, 4113-4115.

16. Johnson D.G., Niemczyk M.P., Minsek D.W., Wiederrecht G.P., Svec W.A., Gaines III G.L., Wasielewski M.R. J. Am. Chem. Soc. 1993, 115, 5692-5701.

17. Zheng G., Pandey R.K., Forsyth T.P., Kozyrev A.N., Dougherty T.J., Smith, K.M. Tetrahedron Lett. 1997, 38, 2409-2412.

18. Pavlov V.Y., Ponomarev G.V. Chem. Heterocycl. Compd. 2004, 40, 393-425.

19. Fischer R., Engel N., Henseler A., Gossauer A. Helv. Chem. Acta. 1994, 77, 1046-1050.

20. Horn S., Dahms K., Senge M.O. J. Porphyrins Phthalocyanines 2008, 12, 1053-1077.

21. Holt A.S., Morley H.V. Can. J. Chem. 1959, 37, 507-514.

22. Oba T., Uda Y., Matsuda K., Fukusumi T., Ito S., Hiratani K., Tamiaki H. Bioorg. Med. Chem. Lett. 2011, 21, 2489-2491.

23. Mathieu J., Panico R. Mécanismes Réactionnels en Chimie Organique. Paris: Hermann, 1972, 765 p.

24. Waters W.A. Mechanisms of Oxidation of Organic Compounds. New-York: John Wiley and Sons, 1964. 152 p.

25. Ma L., Dolphin D. Tetrahedron 1996, 52, 849-860.

26. Smith K.M., Goff D.A., Simpson D.J. J. Am. Chem. Soc. 1985 , 107, 4946-4954.

27. Belykh D.V, Tarabukina I.S., Gruzdev I.V., Kodess M.I., Kutchin A.V. J. Porphyrins Phthalocyanines. 2009, 13, 949956.

28. Belykh D.V., Kopylov E.A., Gruzdev I.V., Kuchin A.V. Russ. J. Org. Chem. 2010, 46, 577-585.

29. Belykh D.V., Buravlev E.V., Mal'shakova M.V., Parshukova N.N., Kopylov E.A., Gruzdev I.V., Kuchin A.V. Chem. Nat. Compd. 2011, 47, 85-90.

30. Tamiaki H., Miyata S., Kureishi Y., Tanikaga R. Tetrahedron 1996, 52, 12421-12432. 